\title{
CONFIRMATION OF THE PLANET AROUND HD 95086 BY DIRECT IMAGING*
}

\author{
J. Rameau ${ }^{1}$, G. Chauvin ${ }^{1}$, A.-M. Lagrange ${ }^{1}$, T. Meshitat ${ }^{2}$, A. Boccaletti ${ }^{3}$, S. P. Quanz ${ }^{4}$, \\ T. Currie ${ }^{5}$, D. Mawet ${ }^{6}$, J. H. Girard ${ }^{6}$, M. Bonnefoy ${ }^{7}$, And M. Kenworthy ${ }^{2}$ \\ ${ }^{1}$ UJF-Grenoble 1/CNRS-INSU, Institut de Planétologie et d'Astrophysique de Grenoble (IPAG) UMR 5274, \\ Grenoble F-38041, France; julien.rameau@obs.ujf-grenoble.fr \\ ${ }^{2}$ Leiden Observatory, Leiden University, P.O. Box 9513, 2300 RA Leiden, The Netherlands \\ ${ }^{3}$ LESIA, Observatoire de Paris, CNRS, University Pierre et Marie Curie Paris 6 and University Denis Diderot Paris 7, 5 place Jules Janssen, F-92195 Meudon, France \\ ${ }^{4}$ Institute for Astronomy, ETH Zurich, Wolfgang-Pauli-Strasse 27, 8093 Zurich, Switzerland \\ ${ }^{5}$ Department of Astronomy and Astrophysics, University of Toronto, 50 St. George St., Toronto, Ontario M5S 1A1, Canada \\ ${ }^{6}$ European Southern Observatory, Casilla 19001, Santiago 19, Chile \\ ${ }^{7}$ Max Planck Institute für Astronomy, Königsthul 17, D-69117 Heidelberg, Germany \\ Received 2013 October 2; accepted 2013 October 24; published 2013 December 6
}

\begin{abstract}
VLT/NaCo angular differential imaging at $L^{\prime}(3.8 \mu \mathrm{m})$ revealed a probable giant planet comoving with the young and early-type HD 95086, also known to harbor an extended debris disk. The discovery was based on the proper motion analysis of two datasets spanning 15 months. However, the second dataset suffered from bad atmospheric conditions, which limited the significance of the re-detection at the $3 \sigma$ level. In this Letter, we report new VLT/NaCo observations of HD 95086 obtained on 2013 June 26 and 27 at $L^{\prime}$ to recover the planet candidate. We unambiguously re-detect the companion HD $95086 \mathrm{~b}$ with multiple independent pipelines at a signal-to-noise ratio greater than or equal to 5. Combined with previously reported measurements, our astrometry decisively shows that the planet is comoving with HD 95086 and inconsistent with a background object. With a revised mass of $5 \pm 2$ Jupiter masses, estimated from its $L^{\prime}$ photometry and "hot-start" models at $17 \pm 4 \mathrm{Myr}$, HD $95086 \mathrm{~b}$ becomes a new benchmark for further physical and orbital characterization of young giant planets.
\end{abstract}

Key words: instrumentation: adaptive optics - planets and satellites: detection - stars: individual (HD 95086)

Online-only material: color figures

\section{INTRODUCTION}

We reported in Rameau et al. (2013) the discovery of a probable 4-5 $M_{\text {Jup }}$ giant planet at $\sim 56 \mathrm{AU}$ (projected separation) from its host-star HD 95086 (A8, $90.4 \mathrm{pc})$. The star belongs to the $17 \pm 4$ Myr old Lower Centaurus Crux association (Pecaut et al. 2012; Meshkat et al. 2013a). Based on two observing epochs acquired in 2012 January and 2013 March, we showed that the detected point-source was likely comoving with HD 95086. However, the confirmation remained ambiguous owing to poor re-detection at the $3 \sigma$ level in 2013 March. Additional observations at the $K s$-band $(2.18 \mu \mathrm{m})$, and even more recently in the $H$-band $(1.6 \mu \mathrm{m})$ with Gemini/NICI (Toomey \& Ftaclas 2003) indicate very red colors, $K s-L^{\prime} \geqslant 1.2 \mathrm{mag}$ and $H-L^{\prime} \geqslant 3 \mathrm{mag}$ (Rameau et al. 2013; Meshkat et al. 2013a). These colors are compatible with a cool and dusty planetary atmosphere. This allowed us to further reject contamination by a background source. The apparent flux and upper limit of the colors for the companion $L^{\prime}$ are compatible with the predictions of the "hot-start" DUSTY evolutionary models (Chabrier et al. 2000) whose color and absolute predictions were recomputed using BT-Settl atmospheric models (Allard et al. 2012) for a mass below $5 M_{\text {Jup }}$.

We re-observed the system on June 26 and 27 using VLT/NaCo with the aim of re-detecting HD 95086 b with a high level of confidence and to constrain its proper motion relative to HD 95086.

\footnotetext{
* Based on observations collected at the European Organisation for Astronomical Research in the Southern Hemisphere, Chile, under programs number 291.C-5023.
}

\section{OBSERVING STRATEGY AND IMAGE PROCESSING}

\subsection{Observations}

To optimize the detection of the faint signal around HD 95086 and to perform high-precision relative astrometry, we observed the star with exactly the same instrumental set-up as in the discovery observations. VLT/NaCo (Rousset et al. 2003; Lenzen et al. 2003) was used in pupil-tracking mode to enable ADI. The observations were carried out at $L^{\prime}$-band $(\lambda=3.8 \mu \mathrm{m}$, $\Delta \lambda=0.62 \mu \mathrm{m})$, with the $\mathrm{L} 27$ camera $\left(\simeq 27.1 \mathrm{mas}_{\text {pixel }}{ }^{-1}\right)$ with $2 \times$ NDIT (number of frames) short $(0.2 \mathrm{~s})$ exposures at each of the four-dither position. The faintness of the target as well as the high airmass prevented us from obtaining saturated exposures to achieve a higher dynamical range. A short set of unsaturated exposures using a neutral density filter (attenuation of $4.36 \pm 0.1 \mathrm{mag}$ ) was taken at the beginning of the observing sequence (PSF). The FWHM measured on the point spread function (PSF) was of $\sim 3.5$ pixels.

Data were acquired on June 26 and 27. The observing set up and conditions are detailed in Table $1 .^{8}$ On June 26, although the conditions were stable, the field rotation was $12^{\circ}$, which corresponds to a rotation of only 1.3 FWHM at the expected projected separation of the signal, i.e., $\sim 620$ mas. On June 27, the amplitude of the field rotation was increased (26.7) but the conditions were slightly less stable.

The astrometric binary IDS 1307 (van Dessel \& Sinachopoulos 1993) was observed on July 7 in field-tracking mode to calibrate the instrument platescale and orientation. IDS 1307 was recalibrated on the $\theta_{1}$ Ori $C$ field (used in 2012 January and

\footnotetext{
8 We also recall the log of the two first datasets in 2012 January and 2013 March.
} 
Table 1

Observing Log of HD 95086 with VLT/NaCo

\begin{tabular}{|c|c|c|c|c|c|c|c|c|c|}
\hline Date & Cam./Filter & $\begin{array}{c}\mathrm{DIT} \times \mathrm{NDIT} \\
(\mathrm{s})\end{array}$ & $N_{\exp }$ & $\pi$-start/end & $\begin{array}{c}(\mathrm{deg}) \\
\langle\text { Airmass }\rangle^{\mathrm{a}}\end{array}$ & $\begin{array}{c}\langle\varpi\rangle^{\mathrm{a}} \\
\left({ }^{\prime \prime}\right)\end{array}$ & $\begin{array}{l}\left\langle\tau_{0}\right\rangle^{\mathrm{a}} \\
(\mathrm{ms})\end{array}$ & $\begin{array}{c}\left\langle E_{\mathrm{c}}\right\rangle^{\mathrm{a}} \\
(\%)\end{array}$ & Ref. \\
\hline 2012 Jan 11 & $\mathrm{~L} 27 / L^{\prime}+\mathrm{ND}$ & $0.2 \times 80$ & 10 & $-9.32 /-8.19$ & 1.39 & 0.75 & 3.6 & 61 & Rameau et al. (2013) \\
\hline 2012 Jan 11 & $\mathrm{~L} 27 / L^{\prime}$ & $0.2 \times 100$ & 156 & $-7.59 / 16.96$ & 1.39 & 0.76 & 3.5 & 58 & Rameau et al. (2013) \\
\hline 2013 Mar 14 & $\mathrm{~L} 27 / L^{\prime}$ & $0.2 \times 100$ & 162 & $3.20 / 28.18$ & 1.41 & 1.77 & 1.0 & 37 & Rameau et al. (2013) \\
\hline 2013 Mar 14 & $\mathrm{~L} 27 / L^{\prime}+\mathrm{ND}$ & $0.2 \times 80$ & 10 & $29.61 / 30.68$ & 1.44 & 1.65 & 0.9 & 32 & Rameau et al. (2013) \\
\hline 2013 Jun 26 & $\mathrm{~L} 27 / L^{\prime}+\mathrm{ND}$ & $0.2 \times 80$ & 10 & $41.0 / 42.0$ & 1.50 & 1.00 & 3.1 & 54 & This work \\
\hline 2013 Jun 26 & $\mathrm{~L} 27 / L^{\prime}$ & $0.2 \times 100$ & 96 & $42.5 / 55.3$ & 1.55 & 1.08 & 2.8 & 45 & This work \\
\hline 2013 Jun 27 & $\mathrm{~L} 27 / L^{\prime}$ & $0.2 \times 80$ & 10 & $28.0 / 29.1$ & 1.44 & 1.17 & 1.4 & 28 & This work \\
\hline 2013 Jun 27 & $\mathrm{~L} 27 / L^{\prime}$ & $0.5 \times 100$ & 186 & $29.6 / 58.9$ & 1.53 & 1.02 & 1.6 & 47 & This work \\
\hline
\end{tabular}

Notes. "ND" refers to the NaCo ND_Long filter (transmission of $\simeq 1.79 \%$ ), "DIT" to exposure time, and $\pi$ to the parallactic angle at start and end of observations.

a The airmass, the seeing $\varpi$, the coherence time $\tau_{0}$, and the coherent energy $E_{\mathrm{c}}$ are estimated in real time by the adaptive-optics system and averaged here over the observing sequence.

2013 March) thanks to contemporaneous observations of both fields obtained in 2012 January.

\subsection{Data Reduction and Analysis}

In order to avoid systematics or biases from image processing and ensure a robust detection, five independent pipelines were used to reduce the data. They are described in detail in Boccaletti et al. (2012; hereafter LESIA), Currie et al. (2012; hereafter A-LOCI), Meshkat et al. (2013b; hereafter Leiden), Amara \& Quanz (2012; PynPoInT), and Lagrange et al. (2010)/Chauvin et al. (2012; IPAG-ADI).

Each pipeline processed the data in a similar way for the first steps (flat-fielding, bad/hot pixel removal, sky-subtraction, registration, and frame selection) to create a mastercube of the individual frames together with the list of associated parallactic angles. Several frames affected by bright waffle modes and bright spiders were also rejected.

The main differences between the pipelines reside in the way the stellar-halo is estimated and subtracted from the mastercube, by using different ADI flavors and different set of parameters. Standard ADI algorithms were applied and we show the results of classic ADI (cADI) and smart ADI (sADI; Marois et al. 2006) applied with IPAG-ADI, Adapted Locally Optimized Combination of Images (A-LOCI; Currie et al. 2012; adapted from Lafrenière et al. 2007), and the most recent principal component analysis (PCA)-based methods (Amara \& Quanz 2012; Soummer et al. 2012) applied with Leiden, LESIA, and PynPoInT. Finally, the residual frames were aligned with true-north to the vertical and combined by mean averaging.

The different ADI techniques produce a high variety of residual images with different speckle intensities and distributions (see Figure 1). This difference also affects the planet's photometry. Using the different pipelines helped to overcome the possible biases related to each algorithm.

In the IPAG-ADI pipeline, the astrometry and photometry were derived as in the discovery paper, following the injection of artificial planets as described in Lagrange et al. (2010); Chauvin et al. (2012). In the remaining pipelines, the astrometry was estimated by fitting the planet's signal with a two-dimensional Gaussian or Moffat function. The systematic bias between the two methods was estimated to be less than 0.7 pixels using the IPAG pipeline.

The main errors on the position of the companion came from the intrinsic measurement of the source position $(0.4$ pixels measured by injecting 10 artificial planets at the same separation as the companion but different position angles and estimating the effect of the surrounding residuals and procedures), the star position ( 0.1 pixels estimated from a series of tests with random shifting of the mastercube and registration as done with the IPAG-ADI pipeline), and finally the astrometric calibration ( 0.1 pixels). The quadratic sum of the error sources lead to an uncertainty of 0.42 pixels. The point-source photometric errors resulted from the uncertainties on the measurements of the signal brightness ( 0.7 mag estimated as for the position), the PSF variability (0.3 mag), and the neutral density transmission (0.1 mag). Added quadratically, we ended up with 0.8 mag of uncertainty.

The signal-to-noise ratio $(\mathrm{S} / \mathrm{N})$ was computed following the same approach as in Rameau et al. (2013). The noise-per-pixel was derived from the standard deviation computed in a ring of 1-FWHM width, centered on the star, with a radius equal to the planet-star separation. The planet was also masked within the ring to compute the noise. The flux of the planet was integrated over an aperture 1-FWHM in diameter. The final integrated $\mathrm{S} / \mathrm{N}$ was computed on the same aperture size considering the noise-per-pixel and aperture size in pixels for renormalization. We tested changing the size of both aperture and coronae to check the effect on the S/N. All measurements were consistent with the ones from the 1-FWHM adopted value. Although there is probably no optimal way to estimate the $\mathrm{S} / \mathrm{N}$ in the speckle dominated regime, the same method was applied to each pipeline and each dataset. However, the purpose is not to directly compare the pipelines with their $\mathrm{S} / \mathrm{N}$ values but rather strengthen the detection.

\section{AN UNAMBIGUOUS COMOVING COMPANION AROUND HD 95086}

\subsection{Re-detection}

In the 2013 June 26 data, we were able to marginally redetect the signal with all algorithms and pipelines (see cADI example in Figure 1, top-left, with a $\mathrm{S} / \mathrm{N}$ of between two and four). No other bright point source is seen in the residual maps. Nonetheless, given the low $\mathrm{S} / \mathrm{N}$, this dataset alone does not allow a firm confirmation of the planet.

We therefore focus our analysis on the data and outputs from the 2013 June 27 observations. Only this dataset is used in the following analysis. Details of the algorithm parameters are provided in Table 2 . 

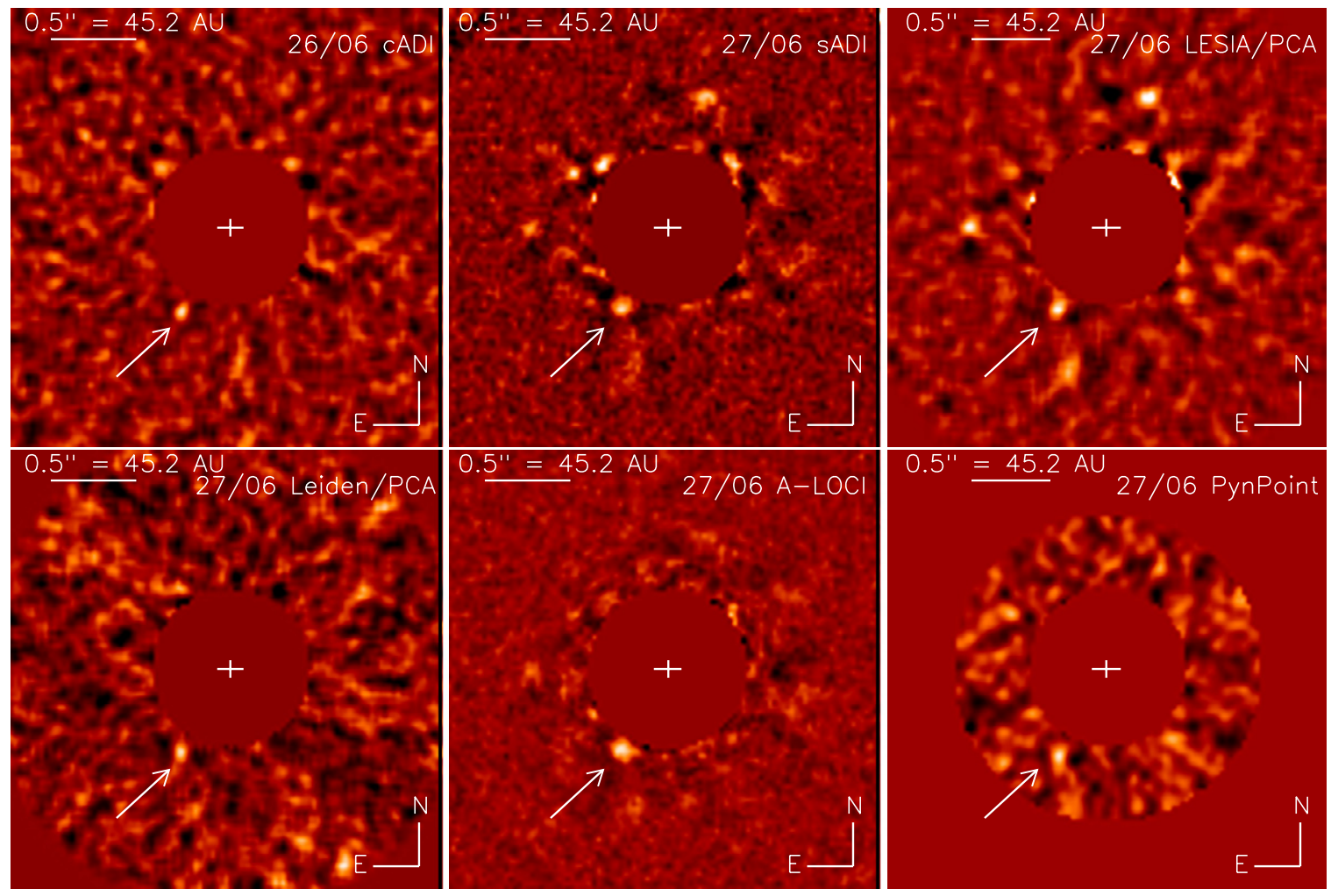

Figure 1. Residual maps of VLT/NaCo images at $L^{\prime}$-band, revealing HD $95086 \mathrm{~b}$ at southeast (arrow). A direct comparison between the pipelines through the $\mathrm{S} / \mathrm{N}$ is not valid due the different noise distributions which are produced. The planet PSFs also appear to be of different sizes due to different cuts and different levels of self-subtraction. Top-left: IPAG-cADI reduction from June $26 ; \mathrm{S} / \mathrm{N} \simeq 4$ due to the small field rotation but good stability. Top-central: IPAG-sADI reduction from June $27 ; \mathrm{S} / \mathrm{N} \simeq 7$. Speckles at northeast and northwest are strong spike-residuals but at a different separation from the central star than the planet. Top-right: PCA reduction following Boccaletti et al. (2012) using seven coefficients over 534; $\mathrm{S} / \mathrm{N} \simeq 6$. Bottom-left Adapted-PCA from Meshkat et al. (2013b) using 16 coefficients over 185, $\mathrm{S} / \mathrm{N} \simeq 5$. Bottom-central: A-LOCI from Currie et al. (2012) where the source is masked over a box of 10 pixels in width; $\mathrm{S} / \mathrm{N} \simeq 13$. Bottom-right: PYNPOINT (Amara \& Quanz 2012) using 40 coefficients over 15,172, $\mathrm{S} / \mathrm{N} \simeq 7.5$. Note PyNPoINT doubles the sampling resolution but it has been rescaled to normal for display purposes. (A color version of this figure is available in the online journal.)

Table 2

ADI Algorithms and Associated Parameters on the Reduction of the 2013 June 27 Data

\begin{tabular}{lccc}
\hline \hline Algorithm & Parameters & S/N & Ref. \\
\hline IPAG/sADI & $r=600$ mas, $N_{\delta}=1$ (FWHM), depth $=6$ frames & 7 & Lagrange et al. (2010) \\
LESIA/PCA & 7 modes out of 534 & 6.5 & Boccaletti et al. (2012) \\
Leiden/PCA & 15 modes out of 185 & 5 & Meshkat et al. (2013b) \\
A-LOCI & $N_{\delta}=0.7$ (FWHM) $g=1, d r=11, N_{A}=35, r_{\text {corr }}=0.16$ & 13 & Currie et al. (2012) \\
PYNPoINT & 40 coefficients out of 15172 & 7.5 & Amara \& Quanz (2012) \\
\hline
\end{tabular}

Note. The S/N cannot be used to directly compare each pipeline and reduction algorithm since the distribution and level of the noise is different in each case.

Figure 1 displays the residual maps with the recovery of HD 95086 b. Some speckles have a high intensity but the planet's signal (southeast) is the only one which systematically appears in each pipeline and ADI-flavor. The planet's signal may look different because, firstly, it is being self-subtracted to different levels, and secondly, different flux levels are adopted in each image.

All the five independent pipelines recover the planet's signal at the expected position with a $\mathrm{S} / \mathrm{N}$ higher than five and using the same method for the $\mathrm{S} / \mathrm{N}$ calculation on the final processed images. The $\mathrm{S} / \mathrm{N}$ variations between the pipelines are related to the different algorithms used for the PSF subtraction and therefore to the different level and distribution of residuals in the final images. In all cases, the signal is unambiguously detected and confirms the recovery of HD $95086 \mathrm{~b}$ in our 2013 June data. As an additional check, with the IPAG-ADI
Table 3

Relative Astrometry and Photometry of HD 95086 b and the Background Source (Background Star)

\begin{tabular}{lccc}
\hline \hline Date & $\begin{array}{c}\text { Sep. } \\
(\mathrm{mas})\end{array}$ & $\begin{array}{c}\text { P.A. } \\
(\mathrm{deg})\end{array}$ & $\begin{array}{c}\Delta L^{\prime} \\
(\mathrm{mag})\end{array}$ \\
\hline \multicolumn{4}{c}{ bkg star } \\
\hline 2012 Jan 11 & $4540 \pm 15$ & $319.03 \pm 0.25$ & $6.2 \pm 0.2$ \\
2013 Mar 14 & $4505 \pm 16$ & $319.42 \pm 0.26$ & $6.1 \pm 0.2$ \\
2013 Jun 27 & $4480 \pm 14$ & $319.52 \pm 0.25$ & $6.0 \pm 0.3$ \\
\hline \multicolumn{4}{c}{ HD 95086 b } \\
\hline 2012 Jan 11 & $624 \pm 8$ & $151.8 \pm 0.8$ & $9.79 \pm 0.40$ \\
2013 Mar 14 & $626 \pm 13$ & $150.7 \pm 1.3$ & $9.71 \pm 0.56$ \\
2013 Jun 27 & $600 \pm 11$ & $150.9 \pm 1.2$ & $9.2 \pm 0.8$ \\
\hline
\end{tabular}



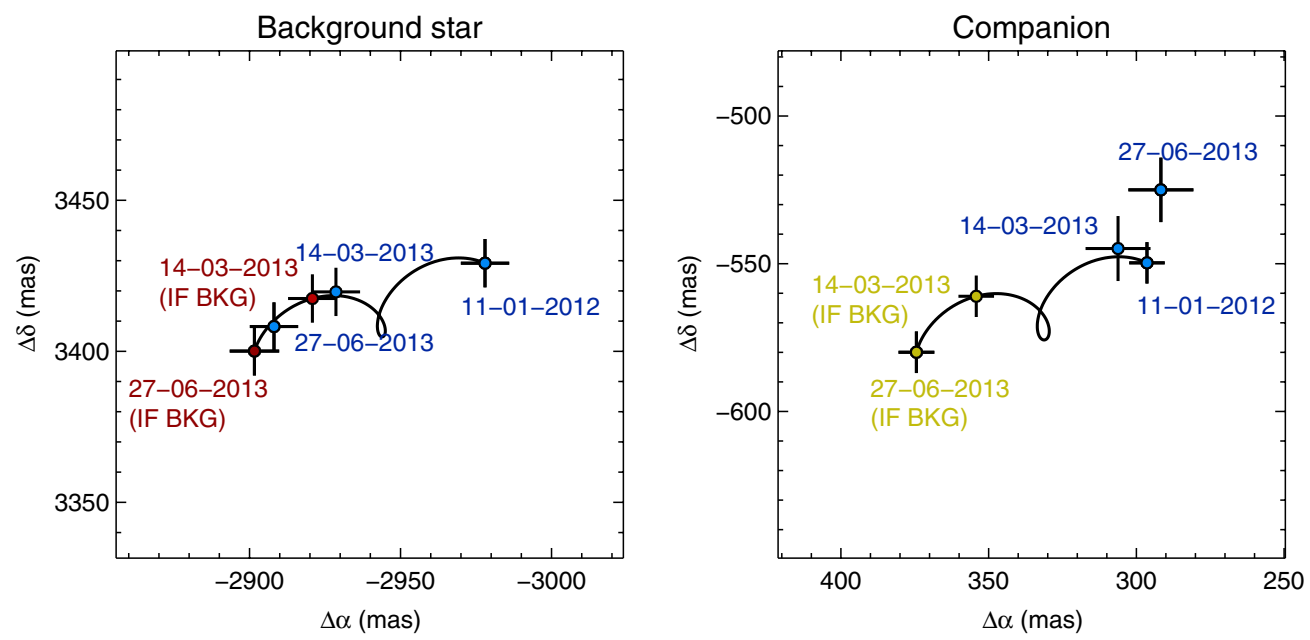

Figure 2. Relative separations between the central star and the companion or background source, in right ascension $(\alpha)$ and declination $(\delta)$. The position measured on 2013 June 27 is over plotted in blue, and the expected position, if the point-source is a fixed background object, is plotted in yellow/red. Previous measurements from Rameau et al. (2013) are also reported. Left: case of the background star. Right: case of HD $95086 \mathrm{~b}$. The 2013 June 27 position lies very close to previous positions and strongly differs from the expected position of a fixed background object.

(A color version of this figure is available in the online journal.)

pipeline, we injected artificial planets in the raw data at the same separation but different position angles and assuming the brightness measured in the following section. Each fake planet was detected in the residual maps generated with the same $\mathrm{S} / \mathrm{N}$ as HD 95086 b. Moreover, the probability that the signal is a residual speckle is very low since the explored parallactic angles strongly differ from one dataset to another between the different epochs: [-7.6; 17] deg in 2012 February 11, [3.2; 28.2] deg in 2013 April 14, and [29.6; 58.9] deg in 2013 June 27.

Hence, a real physical object is re-detected with a good confidence level from the latest dataset.

\subsection{Astrometry and Photometry}

Since the first two measurements of the probable planet were done within sADI residual maps with the IPAG-ADI pipeline, we used the same reduction algorithm to characterize the present dataset. We estimated a separation of $600 \pm 11$ mas and a position angle of $150.9 \pm 1.2$ (see Table 3 ). The other pipelines give similar values, all consistent within 20 mas and $1^{\circ}$. Figure 2 shows the relative position of the star with respect to HD 95086 from each epoch at $L^{\prime}$, and also the track and positions assuming a fixed background object. The position of the companion lies in the same region as the first two epochs and strongly excludes a background object with a $\chi^{2}$ probability of $10^{-16}$. The pointsource is thus comoving with the star.

As was done in the discovery paper, we used the $4^{\prime \prime} .5$ background star visible in the field-of-view to assess our astrometric measurements. Using Moffat-fitting on co-added (no ADI processing) residual images, we estimated a separation of $4^{\prime \prime} .480 \pm 0$. .'014 and a position angle of $319.52 \pm 0.25$. Our original measurements from 2012 January and 2013 March, showing that the point source is a stationary background object, are corroborated by the third point in Figure 2, which lies very close to the expected position of 2013 June. Therefore, the background star confirms our ability to assess or exclude background behavior with high precision.

The photometric measurement on HD $95086 \mathrm{~b}$ is $\Delta L^{\prime}=$ $9.2 \pm 0.8 \mathrm{mag}$, consistent in all pipelines and with previous estimates.

\section{CONCLUSIONS AND PROSPECTS}

New observations of HD 95086 were carried out with exactly the same instrumental set-up as the discovery paper, namely at $L^{\prime}$-band with VLT/NaCo in the ADI mode. We applied five different, independent pipelines to reduce the data. Each of them led to the confirmation of the point-source to the southeast of the star. Precise astrometric measurements showed the signal is not a background object with a probability of $10^{-16}$. HD $95086 \mathrm{~b}$ is therefore a companion comoving with its host-star.

From the three set of data, we revised the mass and projected separation of the planet. With $\Delta L^{\prime}=9.79 \pm 0.40 \mathrm{mag}$ in 2012 January, $9.71 \pm 0.56$ in 2013 March and $9.2 \pm 0.8$ in 2013 June, we estimate that its absolute magnitude is $M_{L}=11.5 \pm 1.1 \mathrm{mag}$. From the BT-Settl models (Allard et al. 2012) which have been shown to be consistent with the red colors of the planet (Meshkat et al. 2013a), the luminosity corresponds to a mass of $5 \pm 2 M_{\text {Jup }}$ at $17 \pm 4$ Myr. We recall that "warm-start" models (Spiegel \& Burrows 2012; Marleau \& Cumming 2013) might predict a higher mass (we could only derive a lower limit of $3 M_{\text {Jup }}$; see Rameau et al. 2013). With a predicted effective temperature of $1000 \pm 200 \mathrm{~K}$, a surface gravity of $3.85 \pm 0.5 \mathrm{dex}$, and very red colors, HD $95086 \mathrm{~b}$ has a cool and dusty atmosphere where the effects of possible non-equilibrium chemistry, reduced surface gravity, and methane bands in the near infrared might be explored in the future. Follow-up observations at different wavelengths, out-of-reach for current facilities, allow characterization of its atmosphere.

With a separation of $623.9 \pm 7.4$ mas in 2012 January, $626.1 \pm 12.8$ mas in 2013 March, and $600 \pm 11$ mas in 2013 June, the projected distance to the host-star is $55.7 \pm 2.5$ AU. Recently, Moór et al. (2013) published resolved Herschel images of a debris disk surrounding the star, extending out to $270 \mathrm{AU}$, with a possible inclination of about $25^{\circ}$. Based on multi-wavelength observations, they built the spectral energy distribution and adopted, as a best-model, a two-component disk at 6 and 64 AU. However, at this stage of modeling, it is not certain whether the disk is best represented by a single 
component model or this specific two-component model. If the gap size and positions of the rings are real, then we can study the properties and influence of HD $95086 \mathrm{~b}$ on the disk structure. The physical distance of HD $95086 \mathrm{~b}$ would be about $61.5 \mathrm{AU}$, very close to the radius of the outer-cold belt. It might sculpt the inner edge of the belt if its chaotic zone (Wisdom 1980) overlaps the belt. Assuming the case of a circular orbit for the planet and planetesimals, Wisdom (1980) showed that the ring inner edge is located at a separation from the planet $\delta a$ according to the relation $\delta a / a=1.3\left(M_{p} / M_{s}\right)^{2 / 7}$, where $a$ is the semi-major axis of the orbit of the planet, $M_{p}$ its mass, and $M_{s}$ the host star mass. The relation is satisfied within the uncertainties of all parameters. Therefore, HD $95086 \mathrm{~b}$ might be responsible for the inner edge of the outer belt. Further orbital monitoring will provide information on the planet's orbital eccentricity and may support this hypothesis if the eccentricity is very small. However, the planet cannot sustain the whole gap alone since its chaotic zone is too small to reach the inner belt at $6 \mathrm{AU}$. The presence of additional planets within the gap is required, as for HR 8799 (e.g., Su et al. 2009). In the discovery paper (Rameau et al. 2013), we excluded the presence of any planet more massive than $5 M_{\text {Jup }}$ beyond about $38 \mathrm{AU}$ from our sensitivity limits, assuming the inclination of the disk. One might speculate on the number and characteristics of these additional planets from the Wisdom (1980) relation. Then, at least three planets would be needed around 10, 20, and 35 AU, on circular orbits. Further deep observations of the system with next-generation planet imagers might reveal these closer-in planets and further observations of the disk might constrain its physical characteristics. Nevertheless, these are speculations on how to maintain such a wide gap since we do know neither the true semi-major axis of HD 95086 b nor whether the disk really has two separate belts.

Finally, a possible in situ formation of HD $95086 \mathrm{~b}$ might be explained with a disk instability scenario (Cameron 1978; Boley 2009; Rafikov 2009) or pebble accretion (Lambrechts \& Johansen 2012). If formed closer to the star, the presence of more massive companions would be required to excite meanmotion resonances and thus induce planet-planet scattering (Scharf \& Menou 2009; Veras et al. 2009). Again, future deep observations might reveal these additional close-in planets. Another scenario would be planet-disk interaction (Papaloizou et al. 2007; Crida et al. 2009) through outward migration. These scenarios might also be tested through orbital monitoring showing eccentricity and/or structures in more resolved images of the disk.

We are grateful to Vanessa Bailey, Kate Su, and Amy Bonsor for fruitful discussions. We thank the Director General of ESO for discretionary time that allowed to confirm the planet. J.R., G.C., and A.M.L. acknowledge financial support from the French National Research Agency (ANR) through project grant ANR10-BLANC0504-01.

Facility: VLT:Yepun (NaCo)

\section{REFERENCES}

Allard, F., Homeier, D., Freytag, B., \& Sharp, C. M. 2012, in Atmospheres from Very Low-mass Stars to Extrasolar Planets, ed. C. Reylé, C. Charbonnel, \& M. Schultheis (EAS Publications Series, Vol. 57; Cambridge: Cambridge University Press), 3

Amara, A., \& Quanz, S. P. 2012, MNRAS, 427, 948

Boccaletti, A., Augereau, J.-C., Lagrange, A.-M., et al. 2012, A\&A, 544, A85

Boley, A. C. 2009, ApJL, 695, L53

Cameron, A. G. W. 1978, M\&P, 18, 5

Chabrier, G., Baraffe, I., Allard, F., \& Hauschildt, P. 2000, ApJ, 542, 464

Chauvin, G., Lagrange, A.-M., Beust, H., et al. 2012, A\&A, 542, A41

Crida, A., Masset, F., \& Morbidelli, A. 2009, ApJL, 705, L148

Currie, T., Debes, J., Rodigas, T. J., et al. 2012, ApJL, 760, L32

Lafrenière, D., Marois, C., Doyon, R., Nadeau, D., \& Artigau, É. 2007, ApJ, 660,770

Lagrange, A.-M., Bonnefoy, M., Chauvin, G., et al. 2010, Sci, 329, 57

Lambrechts, M., \& Johansen, A. 2012, A\&A, 544, A32

Lenzen, R., Hartung, M., Brandner, W., et al. 2003, Proc. SPIE, 4841, 944

Marleau, G.-D., \& Cumming, A. 2013, arXiv:1302.1517

Marois, C., Lafrenière, D., Doyon, R., Macintosh, B., \& Nadeau, D. 2006, ApJ, 641,556

Meshkat, T., Bailey, V., Rameau, J., et al. 2013a, ApJL, 775, L40

Meshkat, T., Kenworthy, M., Quanz, S. P., \& Amara, A. 2013b, arXiv:1310.8577

Moór, A., Ábrahám, P., Kóspál, Á., et al. 2013, ApJL, 775, L51

Papaloizou, J. C. B., Nelson, R. P., Kley, W., Masset, F. S., \& Artymowicz, P. 2007, in Protostars and Planets V, ed. B. Reipurth, D. Jewitt, \& K. Keil (Tucson: Univ. Arizona Press), 655

Pecaut, M. J., Mamajek, E. E., \& Bubar, E. J. 2012, ApJ, 746, 154

Rafikov, R. R. 2009, ApJ, 704, 281

Rameau, J., Chauvin, G., Lagrange, A.-M., et al. 2013, ApJL, 772, L15

Rousset, G., Lacombe, F., Puget, P., et al. 2003, Proc. SPIE, 4839, 140

Scharf, C., \& Menou, K. 2009, ApJL, 693, L113

Soummer, R., Pueyo, L., \& Larkin, J. 2012, ApJL, 755, L28

Spiegel, D. S., \& Burrows, A. 2012, ApJ, 745, 174

Su, K. Y. L., Rieke, G. H., Stapelfeldt, K. R., et al. 2009, ApJ, 705, 314

Toomey, D. W., \& Ftaclas, C. 2003, Proc. SPIE, 4841, 889

van Dessel, E., \& Sinachopoulos, D. 1993, A\&AS, 100, 517

Veras, D., Crepp, J. R., \& Ford, E. B. 2009, ApJ, 696, 1600

Wisdom, J. 1980, AJ, 85, 1122 